\title{
A case report of Sino-orbital Mucormycosis in a COVID-19 patient from eastern India: a "Red-alert" to physicians emphasizing the importance of early microbiological diagnosis
}

\author{
${ }^{1 *}$ Nidhi Dawar Pal, ${ }^{2}$ Anirban Pal, ${ }^{3}$ Debi Kundu, ${ }^{3}$ Puspendu Dutta \\ ${ }^{1}$ Consultant Microbiologist, Sankara Netralaya, Kolkata \\ ${ }^{2}$ Apollo Gleneagles Medical Center, Kolkata \\ ${ }^{3}$ Sankara Netralaya, Kolkata
}

\begin{abstract}
A 65 years old diabetic female, presented with swelling of left eye and multiple discharging sinuses near the inner canthus of the left eye. She had a history of recent COVID-19 infection and was treated with steroids. The pus from peri-orbital sinuses, in $\mathrm{KOH}$ stain showed presence of broad, non-septate, right-angle branching fungal filaments. An early microbiological diagnosis of Mucormycosis, based on the direct smear findings substantiated the clinical diagnosis and helped the patient to get timely treatment.We emphasize that a request for an early microbiological diagnosis may affect the outcome in this highly invasive fatal disease.
\end{abstract}

Keywords: Sino-orbital Mucormycosis, COVID-19, microbiological diagnosis

\section{Introduction:}

Mucormycosis is a highly opportunistic fungal infection with fatal consequences. It commonly affects immunocompromised individuals including those with hematological malignancy, diabetes mellitus and organ transplantation. [1] A resurgence of invasive fungal infections like Mucormycosis infection in COVID19 pandemic, is due to extensive pulmonary disease and immune dysregulation associated with the disease which is further exacerbated by indiscriminate use of steroids, monoclonal antibodies and broad-spectrum antibiotics. [2] The concern with

*Author for Correspondence. E-mail: nidhidawarpal@gmail.com

This work is licensed under a Creative Commons Attribution-Noncommercial-Share Alike 4.0 International License. 
Mucormycosis is that it's a rapidly spreading and life-threatening infection.A few cases of sinoorbital Mucormycosis during COVID-19 pandemic had already been reported from different parts of India [3] [4]. Second wave of COVID19 pandemic setting in eastern India, we report a case of sino-orbital Mucormycosis in a COVID-19 patient from Kolkata, India. This case is one of earliest cases diagnosed in this part of the country during second wave of COVID-19. The fatality rate of Mucormycosis is very high and orbital / intracranial involvement or irreversible immune suppression increases it further.[5]The purpose of reporting this case is to alert the physicians to look for Mucormycosis in the immune-compromised COVID-19 patients and request for early microbiological diagnosis. Mucormycosis is difficult to diagnose and only very high suspicion and early aggressive management can prevent the fatal consequences.

\section{Case report:}

A 65 years old female, came for ophthalmology consultation in a tertiary care eye center Kolkata, India. She was from Jharkhand and referred from multiple peripheral centers. She had recent history of COVID19 infection 17 days back, diagnosed by RT-PCR and received steroids for treatment. Her co-morbidities included diabetes mellitus controlled with insulin treatment.

Her chief complaints were facial pain, swelling and inability to open the left eye with associated discharge. As per her past history, she was uneventful with usual treatment till the $10^{\text {th }}$ day of COVID-19 infection. Then she suddenly developed nasal blockade, facial pain followed by blurring of vision and swelling of left eye. The swelling was gradually progressive and slowly the patient was unable to open the left eye. After 2 days of onset of the symptoms, excessive discharge was noted from the eye. On examination, severe left lid edema with extension to left side of face and upper lip was noted. There was proptosis and complete ptosis of left eye with frozen globe. Multiple peri-orbital sinuses discharging pus near inner canthus were also present. Left eye showed no perception of light. On fundus examination the lens was hazy, disc was pale, retina edematous and cherry red spot was visible indicating CRAO (central retinal artery occlusion). Systemic examination and vital signs were within normal limits.

Regarding her investigations: CECT PNS showed left sided proptosis, enhanced mucosal thickening of all the sinuses, more marked in left maxillary antrum and destruction of left anterior ethmoid septae, medial wall of left maxillary antrum, medial wall of the left orbit, superior-most portion of nasal septum, left middle and inferior turbinates and left sphenoid sinus. MRI of brain with GAD+ venography showed mucosal thickening and collection in left maxillary, sphenoidal and ethmoidal sinuses, thinning of left medial orbital wall, left ethmoidal, superior and middle turbinate bones. Extension was noted into left intra-orbital region. There was inflammation of left sided retro-orbital fat, left extra-ocular muscles and enhancement of optic chiasma and cavernous sinus. With these investigations in background and clinical examination, provisional diagnosis of left sided invasive fungal sinusitis with orbital spread was made.

Microbiological diagnosis: The pus from peri-orbital sinus was sent for Gram stain, $\mathrm{KOH}$ stain, $\mathrm{KOH}$ calcofluor white stain, bacterial and fungal culture.Gram stain of pus was negative for bacteria. Direct examination with $\mathrm{KOH}$ (Figure 1) and $\mathrm{KOH}$ calcofluor white under fluorescent 
microscope (Figure 2) showed the presence of plenty of thin walled, broad, non-septate, wide angle irregularly branching fungal filaments.

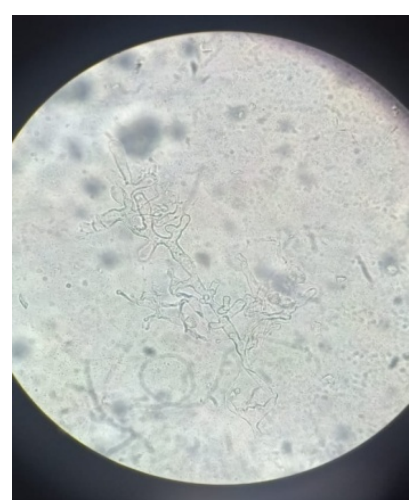

Figure 1: KOH mount showing broad, nonseptate, wide-angled branching hyphae

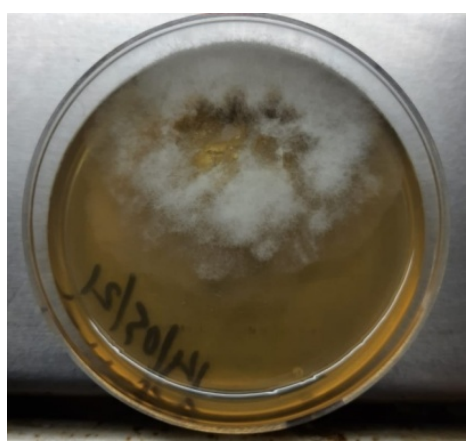

Figure 3:Day 3 growth White cottony colony in SDA at $25^{\circ} \mathrm{C}$

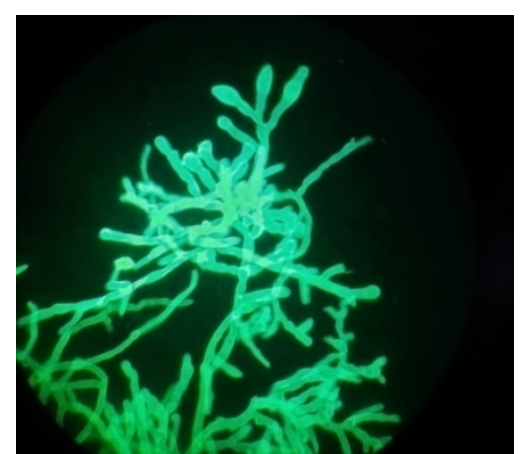

Figure 2: KOH calcofluor white stain showing broad, non-septate, wide-angled branching hyphae under fluorescent microscope.

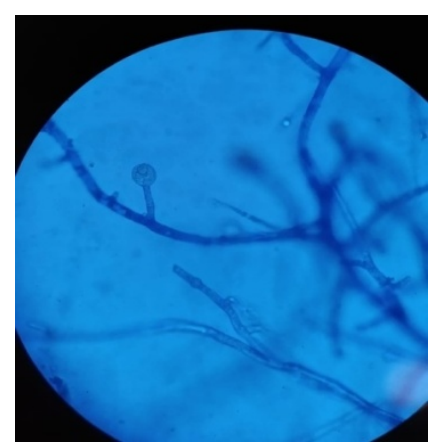

Figure 4: LPCB mount showing small sporangiophore bearing spherical sporangium

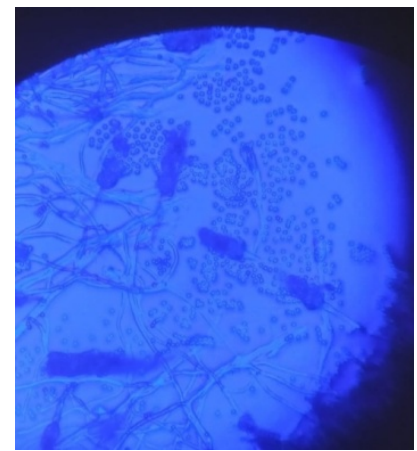

Figure 5: LPCB mount showing bursting of sporangium releasing sporangiospores. 
This direct microscopic examination based diagnosis was communicated to the physician to initiate prompt treatment. The patient was urgently sent to multi-specialty hospital for treatment and further referrals given to ENT surgeon and neurosurgeon. Liposomal Amphotericin B was started and extensive surgery was done by a team of ophthalmic, ENT and neurosurgeon. The patient was saved and was doing well till last communication. The microscopic findings were further confirmed by culture. Cultures were done on SDA and incubated at $25^{\circ} \mathrm{C}$ and on blood agar, brain heart infusion broth incubated at $37^{\circ} \mathrm{C}$, chocolate agar incubated at $37^{\circ} \mathrm{C}$ in $10 \% \mathrm{CO}_{2}$ and Brucella blood agar incubated at $37^{\circ} \mathrm{C}$ under anaerobic conditions. Bacterial cultures were negative after 48 hours of incubation. Anaerobic bacterial culture incubated further for 12 days came out to be negative. On $3^{\text {rd }}$ day of incubation SDA at $25^{\circ} \mathrm{C}$ showed pure growth of white cottony colony (Figure 3). LPCB (lacto phenol cotton blue) mount was done from colony in a biosafety cabinet following all safety precautions. LPCB mount showed presence of broad, nonseptate, right angle branching fungal filaments with small sporangiophores bearing thin walled spherical sporangium. (Figure 4) No rhizoids and no apophysis were seen. After 10 days of growth sporangium burst open releasing spherical sporangiospores (Figure 5) Diagnosis of Mucor species was made based on the culture findings. Fungal PCR could not be done as facility was not available at the center.

\section{Discussion:}

Mucormycosis caused by Mucorales species of the phylum Zygomycota, is one of the most fulminant form of Zygomycosis [6]. In Indian population the prevalence of Mucormycosis is about 80 times higher than the developed countries [7]. Although Mucormycosis is extremely rare in healthy individuals but with the COVID-19 pandemic hitting India, has renewed the interest in this extremely fatal disease. Altered immunity and indiscriminate use of corticosteroids [8] in treatment of COVID-19 infection may be the major predisposing factors. In addition, diabetes mellitus is a common risk factor associated with Mucormycosis in India. [9] With the ongoing COVID-19 pandemic, sporadic cases of invasive sino-orbital Mucormycosis in COVID-19 patients are being reported from different parts of the country. Sen et al, reported a series of six cases from Hyderabad [3]. Sarkar et al reported a cluster of ten cases of orbital Mucormycosis from Puducherry [4].

The "Red alert" with Mucormycosis is the rapid progression and angio-invasion from the nasal and sinus mucosa into the orbit and brain [10]. Rapidity of dissemination in Mucormycosis is an extraordinary phenomenon and even a delay of 12 hours in the diagnosis could be fatal [11]. A delay of even six days in initiating treatment doubles the 30-day mortality from $35 \%$ to $66 \%$ [12]. The intracranial involvement of Mucormycosis increases the fatality rate to as high as $90 \%$ [5]. Although confirmation of diagnosis and species identification is essential, treatment should be initiated as soon as the diagnosis is suspected. Even with early aggressive medical therapy combined with other modalities, the prognosis of Mucormycosis is poor. [12]

In this case, the patient from the neighbouring state faced numerous challenges for her diagnosis and treatment in her state and then was referred to our centre. She visited our centre quite late, 
but thorough clinical examination, high index of suspicion and early microscopic diagnosis, helped her to get the timely treatment. Early diagnosis lead to prompt medical and surgical interventions which saved her life.This case is perhaps an eye-opener. The aggressive progression and the fatality of the disease needs to be re-emphasized in the medical fraternity. The sensitization of the physicians and early microbiological diagnosis is the need of the hour as the incidence of sino-orbital-Mucormycosis is likely to rise in the background of the COVID19 pandemic. Only an early microbiological diagnosis followed by prompt treatment can decrease the mortality and morbidity of this fatal invasive fungal disease.

Conclusion: A high level of clinical suspicion combined with early microbiological diagnosis in immune deficient COVID19 patients of eastern India, can be the key to a better outcome in otherwise fatal Mucormycosis infection.

\section{Acknowledgement: None}

\section{Conflict of interest: None}

\section{References}

1. Hirabayashi KE, Idowu OO, Kalin-Hajdu E, et al. 2019.Invasive Fungal Sinusitis: Risk Factors for Visual Acuity Outcomes and Mortality. Ophthalmic Plast Reconstr Surg. 35(6), pp 535-542.

2. Ismaiel WF, Abdelazim MH, Eldsoky I, et al. 2021The impact of COVID-19 outbreak on the incidence of acute invasive fungal rhinosinusitis. Am J Otolaryngol. 14;42(6), pp 103080.

3. Sen M, Lahane S, Lahane TP, et al . 2021. Mucor in a Viral Land: A Tale of Two Pathogens. Indian J Ophthalmol. 69(2), pp 244-252.

4. Sarkar S, Gokhale, Choudhury T, et al. 2021 COVID-19 and orbital mucormycosis, Indian Journal of Ophthalmology: 69, pp 1002-1004

5. Deutsch P.G., Whittaker J., Prasad S. 2019 Invasive and non-invasive fungal rhinosinusitis - a review and update of the evidence. Medicina. 55, pp 1-14.

6. Sugar A.M. 1992 Mucormycosis. Clin. Infect. Dis. 14, pp S126-S129

7. Chander J., Kaur M., Singla N., et al. 2018 Mucormycosis: battle with the deadly enemy over a fiveyear period in India. J. Fungi. 4(2). pp 46.

8. Skiada A., Pagano L., Groll A. 2011 Zygomycosis in Europe: analysis of 230 cases accrued by the registry of the European confederation of medical Mycology (ECMM) working group on zygomycosis between 2005 and 2007. Clin Microbiol Infect. 17(12), pp 1859-1867.

9. Prakash H., Chakrabarti A. 2019 Global epidemiology of mucormycosis. J Fungi.5. pp 26.

10. Peterson K.L., Wang M., Canalis F.R, et al.1997. Rhinocerebral mucormycosis: evolution of the disease and treatment options. Laryngoscope. 1997;107:855-862

11. Maartens G., Wood M.J. The clinical presentation and diagnosis of invasive fungal infections. J Antimicrob Chemother. 28. pp 17-44.

12. Werthman-Ehrenreich 2021A. Mucormycosis with orbital compartment syndrome in a patient with COVID-19. Am. J. Emerg. Med. 42. pp 264 